\title{
Abordaje de la cesación tabáquica de un paciente desde la farmacia comunitaria. Programa 'CESAR'
}

\author{
Jesús Carlos Gómez-Martínez' ${ }^{1}$ Noelia Tejedor-García ${ }^{2,3}$, Coral García-Pastor ${ }^{2,4}$ \\ 1. Farmacéutico comunitario en Barcelona. 2. Doctora en Farmacia. 3. Farmacéutica comunitaria en Madrid. 4. Farmacéutica comunitaria en Alcalá de \\ Henares (Madrid).
}

\section{PALABRAS CLAVE}

Tabaquismo, cesación tabáquica, servicios profesionales farmacéuticos

\section{ABREVIATURAS}

ENS: Encuesta Nacional de Salud PNT: Procedimiento Normalizado de Trabajo

PRM: problema relacionado con la medicación

\section{KEYWORDS}

Tobacco use disorder, tobacco use cessation, community pharmacy services

\section{Introducción}

El tabaquismo es una epidemia que mata cada año a 7 millones de personas a nivel mundial (1). Según la Encuesta Nacional de Salud (ENS) de 2017 (2), el 22,1 \% de la población mayor de 15 años afirma que fuma a diario y el 2,3 \% ocasionalmente; mientras que según la ENS de 2012 (3) el 24,0 \% de la población afirmaba fumar a diario y el 3,1\% ocasionalmente; esto supone una bajada de 1,9 puntos porcentuales en 5 años en fumadores diarios y un $0,4 \%$ en los ocasionales. Según varios estudios, aproximadamente el $70 \%$ de los fumadores quiere dejar de fumar, pero menos del $10 \%$ alcanza la abstinencia al cabo de un año y solo un $2 \%$ lo consigue si el paciente no ha recibido ningún tipo de intervención (4). Además, según datos de la campaña sin humo, promovida por SEFAC en el último año, el 25,6 \% de los pacientes que desean dejar de fumar acuden a su farmacéutico en su primer intento, el $40 \%$ en el segundo y el 46,6 \% en el tercero (5).

En el año 2013, desde SEFAC se puso en marcha a nivel nacional el programa de Cesación tabáquica desde farmacia comunitaria (CESAR), que consiste en tres partes: un curso teórico, sesiones prácticas, y registro de casos clínicos, incluyendo un test de evaluación y la valoración de los casos registrados en la plataforma de $S E F A C$ e_XPERT, que en su conjunto capacitan para la realización del servicio profesional. Más de 1.500 farmacéuticos hasta la fecha se han registrado a través del programa CESAR y en mayo de 2016 ya se había completado el seguimiento durante 180 días de 380 pacientes (319 estaban abstinentes), unos datos muy superiores a los obtenidos en otras intervenciones realizadas por personal sanitario (6) (7), lo que demostraba las ventajas del programa CESAR y la intervención del farmacéutico en la cesación tabáquica (realizando educación sanitaria, supervisando el tratamiento farmacológico, posibilitando la participación en terapias individuales y colectivas, etc.). Estos datos, junto a las mejoras de salud evidentes que manifiestan los pacientes (mejor percepción de sabores y olores, aumento de la capacidad pulmonar, etc.), deben incentivarnos a la instauración de este servicio de atención farmacéutica.
Financiación: ninguna.

Conflicto de intereses: ninguno.

Cite este artículo como: Gómez-Martínez JC, Tejedor-Garcia N, Garcia-Pastor C. Abordaje de la cesación tabáquica de un paciente desde la farmacia comunitaria. Programa 'CESAR'. Farmacéuticos Comunitarios. 2020 Mar 06;2(1):25-28. doi:10.5672/FC.2173-9218.(2020/Vol12).001.05

Correspondencia: Jesús Carlos Gómez-Martinez (jesuscarlosgm@gmail.com).

ISSN 1885-8619 @SEFAC (Sociedad Española de Farmacia Familiar y Comunitaria). Todos los derechos reservados. 


\section{Presentación del caso}

Hombre de 41 años que acude habitualmente a la farmacia a retirar su medicación; durante la dispensación de sus medicamentos, siguiendo los Procedimientos Normalizados de Trabajo (PNT) recogidos en el documento de consenso de FORO, comenta su preocupación por su estado de ansiedad y refiere que últimamente fuma mucho más debido a esa situación. Se aplica el algoritmo 1 y se observa que está en fase de contemplación, ya que comenta que en aproximadamente 6 meses piensa dejar de fumar, valora ventajas e inconvenientes de hacerlo y durante diferentes visitas a la farmacia comunitaria se refuerza el hecho de que deje de fumar a través de entrega de folletos, recomendaciones, etc.

En una visita posterior se observa que el paciente se encuentra en fase de preparación (en menos de 1 mes estaría dispuesto a dejar de fumar) y en ese momento se le plantea su inclusión en el servicio de cesación tabáquica de la Farmacia Jesús C. Gómez Martinez.

El paciente ha sido consumidor de 20 cigarrillos al día durante 21 años, con un intento previo de cesación tabáquica durante 6 meses en el que empleó parches de nicotina. El motivo de la recaída fue la ansiedad en el trabajo. Es un paciente que suele realizar mucho de- porte, toma una alimentación equilibrada y acude regularmente a la farmacia.

\section{Estudio y evaluación}

El paciente se encuentra inicialmente en fase de contemplación según el modelo de Prochaska y DiClemente pero, después de realizar educación sanitaria y labores de concienciación sobre los perjuicios del tabaco, pasa a fase de preparación y acepta participar en el programa de cesación tabáquica instaurado en la farmacia.

En la visita inicial y tras firmar el consentimiento informado, se incluye en el servicio de cesación tabáquica y todos los datos se registran a través del programa SEFAC e_XPERT. Durante le entrevista inicial se recogen los siguientes datos:

- Presenta una dependencia alta a la nicotina medida mediante el Test de Fagërstrom y una motivación moderada a dejar de fumar medida mediante el Test de Richmond.

- Su historia tabáquica indica un consumo de 21 cigarrillos al día (tras las comidas, en los descansos y en situaciones de estrés). Generalmente los fuma con ansiedad y no suele finalizarlos.

- Sus motivaciones intrínsecas principales son aumentar su capacidad pulmonar y evitar enfermedades asociadas al consumo de tabaco.

- De manera habitual consume: lorazepam $1 \mathrm{mg}$ (2-0-2) y oxacabamazepina $300 \mathrm{mg}$ (1-0-1), detectándose un problema relacionado con la medicación (PRM) debido al mal uso del Lorazepam. Se deriva al médico para un reajuste de la dosis que permite mejorar el estado de ansiedad.

- Los parámetros obtenidos el primer día de consulta fueron: Índice de Masa Corporal $\left(25 \mathrm{~kg} / \mathrm{m}^{2}\right)$, presión arterial PAS/PAD (135/85 mmHg), capacidad pulmonar con el COPD6 (FEV1/FEV6: 0,80) y monóxido de carbono medido mediante carboxímetro (10 ppm).

\section{Intervención}

Una vez incluido en el programa de cesación tabáquica se aplica el algoritmo de intervención farmacéutica y se deriva el paciente al médico, informando del estado de situación en cuanto a la medicación, así como de la entrada en el programa de cesación tabáquica.

Es un paciente que suele realizar mucho deporte, hecho que le ayuda a controlar su estado de ansiedad y su adicción al tabaco. Durante todo el proceso de cesación tabáquica se reforzó este hecho para que le ayudase en su intento de dejar de fumar.

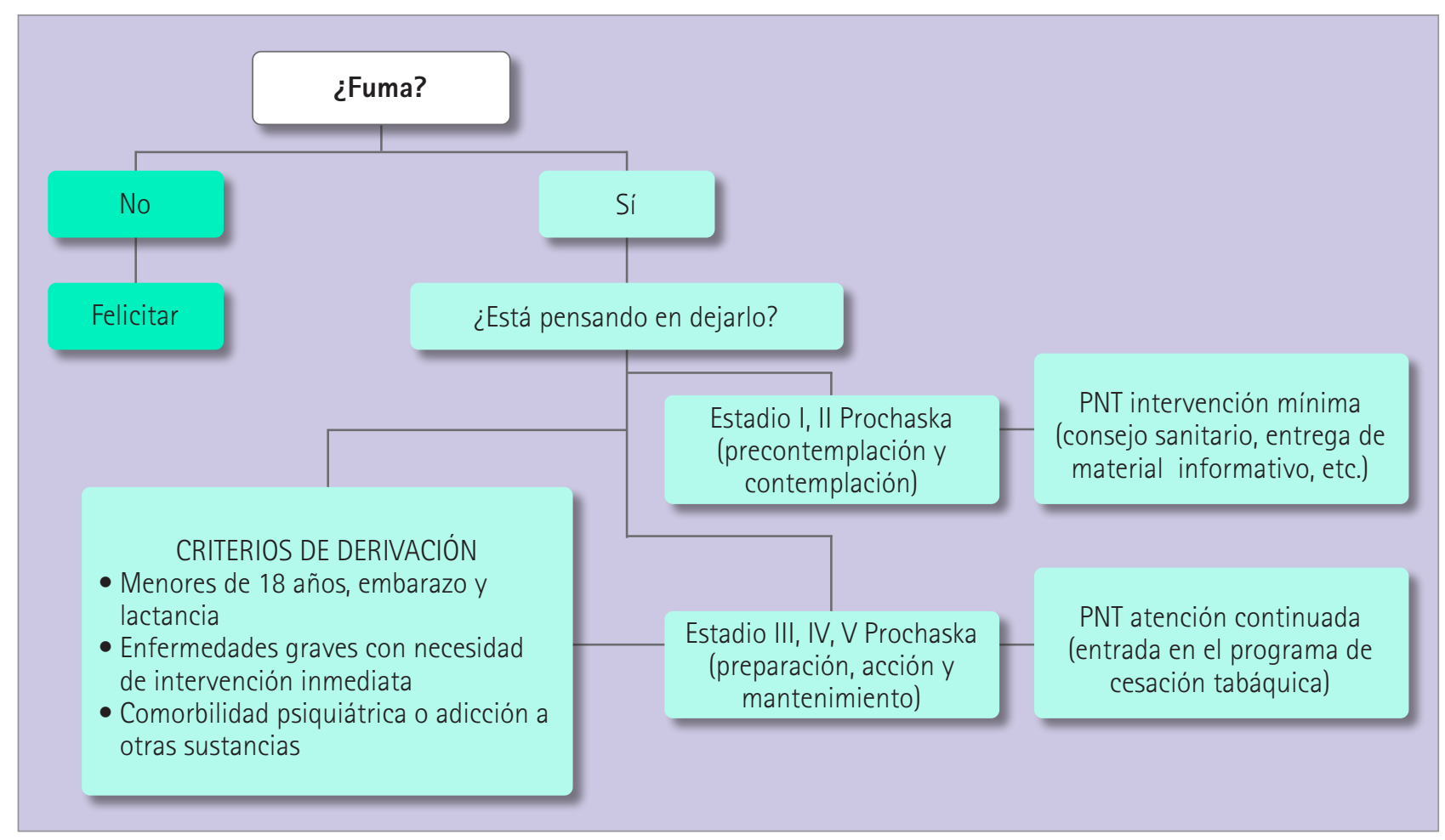

Figura 1 Algoritmo de oferta del servicio profesional de cesación tabáquica (6) 


\section{Persona que quiere dejar de fumar y pide asesoramiento farmacéutico}

Menor de 18 años o mayor de 70 años con patología de base

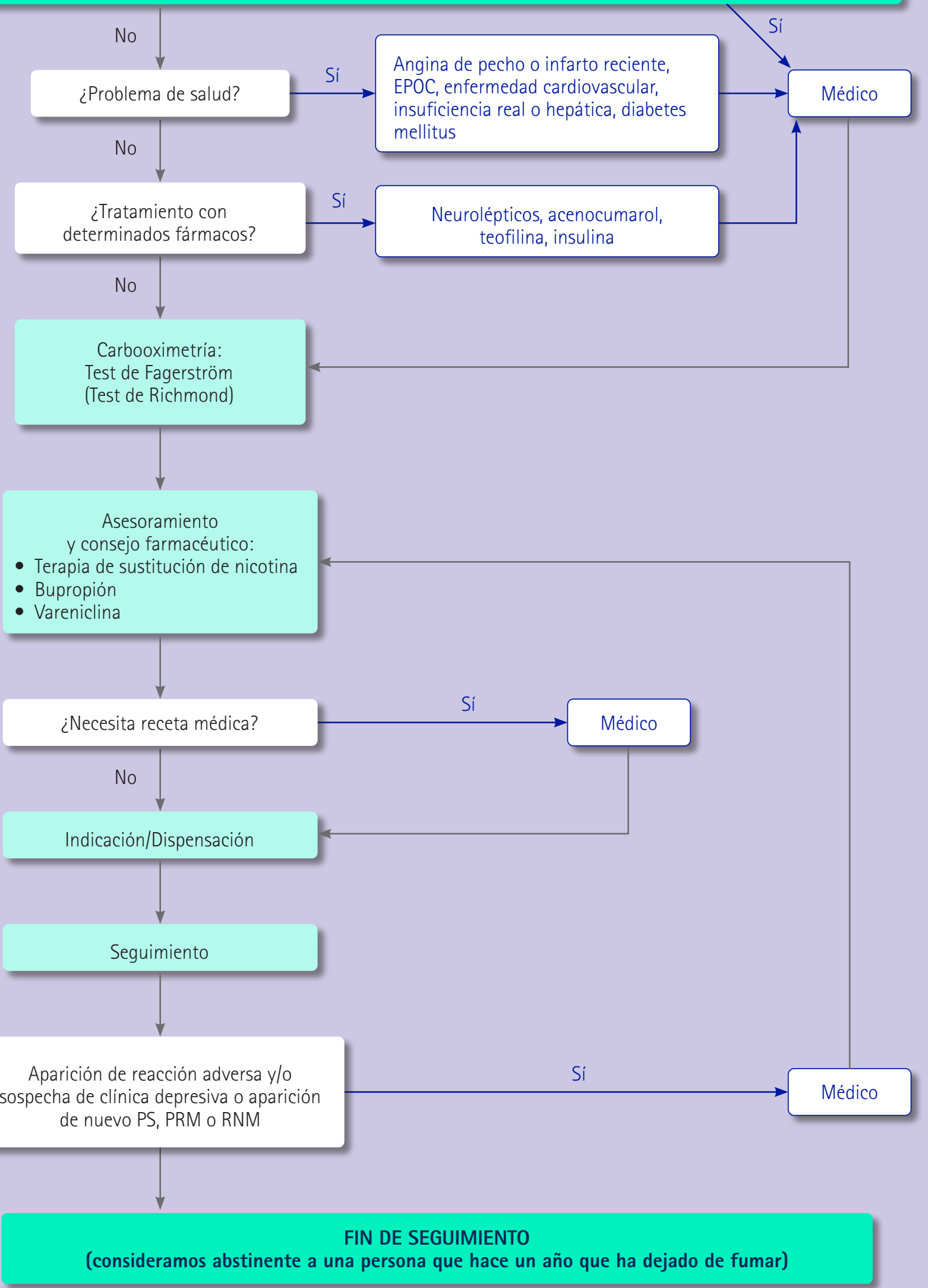

Figura 2 Algoritmo de intervención farmacéutica en cesación tabáquica según modelo CESAR (9) 
Sin embargo, a pesar de sus deseos de mejorar su salud y calidad de vida, el paciente presenta inseguridad a la hora de fijar el día D (día señalado por parte del paciente para iniciar el abandono del tabaco). Finalmente, y tras varias intervenciones por parte del farmacéutico, el paciente decide comenzar el día 1 del mes siguiente.

Dado que el paciente en su anterior intento lo consiguió con terapia sustitutiva de nicotina (parches de nicotina $21 \mathrm{mg} / 24 \mathrm{~h}$ durante 15 días, un tiempo menor del recomendado y motivo por el cual pudo haberse producido la recaída), se decide iniciar con esta pauta e irla ajustando según necesidad.

\section{Resultado y seguimiento}

A los 7 días del día D se realizó la siguiente visita. El paciente no había vuelto a fumar y continuaba con el tratamiento. En esta sesión se felicitó al paciente por el esfuerzo realizado y se dieron consejos de refuerzo y de cómo afrontar situaciones difíciles.

$\mathrm{Al}$ mes se redujo la dosis de los parches de nicotina de $21 \mathrm{mg} / 24 \mathrm{~h}$ a $14 \mathrm{mg} / 24 \mathrm{~h}$ durante el segundo mes, $7 \mathrm{mg} / 24 \mathrm{~h}$ durante 15 dias y, posteriormente, la misma dosis en días alternos. Se mantuvo el refuerzo positivo y se realizó educación nutricional al paciente para la reducción de peso, debido a un aumento moderado.

Tras 7 meses de abstinencia, el paciente tuvo una recaída durante 15 días durante la cual acudió a la farmacia donde se le recordaron, al igual que en las otras sesiones, sus motivaciones intrínsecas y se valoró el progreso realizado antes de la recaída (mejora la capacidad pulmonar, mayor percepción de olores, disminución de la ansiedad...), tras la cual el paciente volvió a dejar de fumar. Desde ese período se efectuaron visitas quincenales y luego mensuales con el fin de reforzar los argumentos por los que el paciente dejo de fumar. A los 6 meses desde la recaída el paciente no fumaba, realizaba deporte y había mejorado su estado de ansiedad. Se felicitó al paciente, se le recordaron los motivos por los que había decidido dejar de fumar y se realizó educación sanitaria. Se derivó al médico para que valorase el estado de ansiedad y la posible retirada del tratamiento debido a la mejora de los síntomas.

$\mathrm{Al}$ año del inicio de las visitas el paciente había dejado de fumar y había disminuido la dosis de su tratamiento para la ansiedad (Lorazepam $0.5 \mathrm{mg}$ a demanda).

\section{Comentario final}

La entrada de este paciente en el programa de cesación tabáquica del servicio CESAR permitió que esta persona dejara de fumar, así como mejorar su estado de ansiedad gracias al abordaje completo del paciente en el que no solo se ha realizado cesación tabáquica, sino que se ha realizado un seguimiento e intervención sobre su medicación. Durante 2 años se realizó su seguimiento para comprobar si estos hábitos se mantuvieron durante el tiempo (hasta un $46 \%$ de los pacientes recae años después de haber abandonado el hábito) (8).

\section{Conclusiones}

El servicio de cesación tabáquica permite afrontar la terapia para dejar de fumar de una forma más eficiente al valorar el estado de salud, medicación, motivos de abandono del tabaco, técnicas de deshabituación, etc.

Los datos sobre el estudio CESAR todavía no han sido publicados, pero basándonos en datos preliminares publicados en el VIII Congreso Nacional de Farmacéuticos Comunitarios, celebrado en mayo de 2018 en Alicante, los resultados parecen alentadores e indican un aumento de la eficacia frente al grupo control, lo que nos debe motivar en la instauración de este programa en la farmacia comunitaria, con el fin de disminuir la incidencia de este problema que nos afecta a todos de manera global al disminuir la esperanza y calidad de vida de nuestros pacientes.
En conclusión, consideramos que es imprescindible destacar la importancia de aplicar los protocolos normalizados de trabajo del servicio de cesación tabáquica, así como publicar los máximos datos posibles sobre su implantación, para demostrar la importancia del farmacéutico comunitario en la cesación tabáquica y en la disminución de los problemas de salud asociados.

\section{Referencias bibliográficas}

1. World Health Organization. WHO report on the global tobacco epidemic, 2017: monitoring tobacco use and prevention policies. Ginebra: WHO; 2017.

2. Instituto Nacional de Estadística. Encuesta Nacional de Salud 2016-2017. España: MSCBS;2018.

3. Instituto Nacional de Estadística. Encuesta Nacional de Salud 2011-2012. España: MSSSI;2013.

4. Laborda MQ, Carreras JM, Sánchez L. Recaída en el abandono del consumo de tabaco: una revisión. Adicciones. 2002 Jan 15;14(1):65-78. doi:10. 20882/adicciones. 514

5. Sociedad Española de Farmacia Comunitaria. Una de cada cuatro personas que quiere dejar de fumar recurre al farmacéutico comunitario en su primer intento. 2019 0ct (Nota de prensa).

6. Guallar-Castillón, P., Lafuente Urdinguio, P., Garteizaurrekoa Dublang, P., Sáinz Martínez, 0., Díez Azcárate, J. I., Foj Alemán, M. y Rodríguez-Artalejo, F. (2003). Probabilidad de éxito en el abandono del tabaco en el curso de dos intervenciones sencillas para dejar de fumar. Revista española de salud pública, 77, 117-124. doi:10.1590/S113557272003000100008

7. Gómez Martínez JC, Mendoza Barbero A, Caelles Franch N, Murillo Fernández MD. Programa CESAR. Capacitación para la prestación del servicio de cesación tabáquica en farmacia comunitaria. Farmacéuticos comunitarios. 2016 May 26;8(Suplemento 1).

8. Agencia de evaluación de Tecnologías sanitarias. Evaluación de la eficacia, efectividad y coste-efectividad de los distintos abordajes terapéuticos para dejar de fumar. Informe de Evaluación de Tecnologías Sanitarias. 2003 Sep 40.

9. Sociedad Española de Farmacia Comunitaria. Procedimiento Normalizado de trabajo del Servicio de Cesación Tabáquica. 2014. 